\title{
Advances in Optimal Detection of Cancer by Image Processing; Experience with Lung and Breast Cancers
}

\author{
Zeinab Mohammadzadeh, Reza Safdari*, Marjan Ghazisaeidi, Somayeh \\ Davoodi, Zahra Azadmanjir
}

\begin{abstract}
Clinicians should looking for techniques that helps to early diagnosis of cancer, because early cancer detection is critical to increase survival and cost effectiveness of treatment, and as a result decrease mortality rate. Medical images are the most important tools to provide assistance. However, medical images have some limitations for optimal detection of some neoplasias, originating either from the imaging techniques themselves, or from human visual or intellectual capacity. Image processing techniques are allowing earlier detection of abnormalities and treatment monitoring. Because the time is a very important factor in cancer treatment, especially in cancers such as the lung and breast, imaging techniques are used to accelerate diagnosis more than with other cancers. In this paper, we outline experience in use of image processing techniques for lung and breast cancer diagnosis. Looking at the experience gained will help specialists to choose the appropriate technique for optimization of diagnosis through medical imaging.
\end{abstract}

Keywords: Cancer - early detection of cancer - image processing - lung neoplasm - breast neoplasm

Asian Pac J Cancer Prev, 16 (14), 5613-5618

\section{Introduction}

Cancer has the highest mortality rate, among other non-communicable diseases in the world (Fallahzadeh et al., 2014). According to Globocan latest report, there are 14.1 million new cancer cases, 8.2 million cancer deaths and 32.6 million people who living with cancer in 2012 worldwide. Almost $57 \%$ (8 million) of new cancer cases, $65 \%$ (5.3 million) of the cancer deaths and $48 \%$ (15.6 million) of the 5-year prevalent cancer cases occurred in the less developed regions (Globocan, 2012a). Therefore, because of high incidence, mortality and burden costs of cancer disease, there is an urgent need for early detection strategies (Mohammadzadeh and Safdari, 2014; Vemuri et al., 2014; Azadmanjir et al., 2015; Shridhar et al., 2015).

In this regard medical images have lots of information about the anatomical structures that are valuable for accurate and early diagnoses, selecting the suitable treatment approach and analyzing treatment outcome (Gao et al., 2003; Dobrescu et al., 2010; Remenyi et al., 2011). Hence, accurate processing and interpreting of medical images, such as a variety of other data in the health care industry, lead to explore the relationship between these data and improvement of clinical performance (Abramoff et al., 2004; Alhadidi et al., 2007; Kannadhasan et al., 2013; G, 2014; Patil et al., 2014). Overall, as defined "Image processing refers to manipulation of the gray level information contained within the pixels of a digital image" (Strickland, 2002). Anyway, because of the human intellectual and visual limitations in accurate image processing, computer-based image processing with image detailed analyzing, and recognizing any abnormal tissue changes can help with early detection of cancer (Giger, 2004; Jain and Vijay, 2013; Hasanabadi et al., 2014). In recent decades, medical image processing has advanced increasingly (He et al., 2012) .

In general, computer-assisted image analysis are used to determining the prognosis and diagnosis diseases, particularly cancers, since the early 1970s (Loukas et al., 2003). Similarly, computerized image processing helps to early and correct detection of malignant tissue and decreases unnecessary biopsies (Polakowski et al., 1997; Ganesan et al., 2013; Sundari et al., 2014). Studies indicated that computer- aided image processing increase cancer detection rates about 20\% (Chen et al., 2012; Kannadhasan et al., 2013). This amount is substantial for decreasing mortality rate. This technique can be used for less experienced physician and learners to cancer diagnosis and correct treatment (Sampat et al., 2005; Rajyalakshmi et al., 2014; Santosh and Sadashivappa, 2014).

The cancer diagnosis with the aid of image processing include following phases: image acquisition, preprocessing (segmentation, enhancement and noise elimination), processing, post processing and diagnosis (Demir and Yener, 2005; He et al., 2012; Patil and Jain, 2014). The preliminary task in processing is eliminating of image 
noise that performed in preprocessing step (Demir and Yener, 2005). In addition, image segmentation is an essential process that used to identify the region of interest (ROI), and critical for content analysis and image understanding (Upadhyay and Wasson; Zhang et al., 2008; Kekre et al., 2010; Ramteke and Jain, 2013). Also, image enhancement improves the images qualities and used to correct image's resolution, reduce the noise of content or highlight its details (Leela and Kumari, 2014). This paper describes some of the image processing techniques that applied to improve and accelerate diagnosis of lung and breast cancers.

\section{Lung Cancer Diagnosis Through Image Processing}

Lung neoplasm which has $12.7 \%$ incidence rate and $18.2 \%$ mortality rate per year (Mukti and Ahmed, 2013) is the most common cancer in the world (Globocan, 2012b; $\mathrm{Kemal}^{1}$ et al., 2014). Studies showed that $80 \%$ of patient with lung cancer died during first five years of diagnosis, and early detection of lung cancer increase survival rate about 10-50 fold (Guo, 2010; Zahir and Mirtalebi, 2012; Kaur et al., 2014). Due to insidious onset, the diagnosis of lung cancer in primary stage is very difficult (Chen et al., 2011); thus image processing through early detection can help increasing the survival rate of lung cancer (Shriwas and Dikondawar, 2015).

Digital image processing can be used for tissue discriminating, lung lesions and nodules detection, types of tumors classifying and tumor growth measurement (Lee et al., 2009) . Image processing for detection of lung cancer has some stages includes image capture, image enhancement, image segmentation and feature extraction. Noise, corruption and interferences of images can be eliminating through the image enhancement techniques.

The next stage, image segmentation has important role in recognition the details of objects in important areas. (Abdulbaki, 2012; Al-Tarawneh, 2012; Tripathy, 2013; Usman et al., 2013).

\section{Experiences in lung cancer diagnosis through image processing}

Bae et al. (2005) developed an automated pulmonary nodule detection program for 20 samples of thin-section multi- detector row CT images of the thorax. The number, size, and location of nodules that detected by the computer program compared with the radiologists' interpretation of images. The results show that nine small nodules were missed in the initial radiologist's reading. Whereas, the overall sensitivity with this technique was $95.1 \%$ for all nodules $3 \mathrm{~mm}$ and larger, for nodules $3 \mathrm{~mm}$ to less than 5 $\mathrm{mm}$, the sensitivity was $91.2 \%$ and For $5 \mathrm{~mm}$ to less than $10 \mathrm{~mm}$ nodules was $97.2 \%$ as well. Therefore, precision of detection through the computer program was higher than radiologist detection for small nodules (Bae et al., 2005).

Whiles the mentioned study focused on the detection of cancerous or non-cancerous nodules in another study by Aoyama et al proposed a computer based technique to differentiate types of nodules (benign or malignant) in chest radiographs samples. To discrimination of radiographs they use the Linear Discriminate Analysis (LDA) and Artificial Neural Network (ANN). In comparing with manual discrimination, LDA had an area under the curve (AUC) value of $88.6 \%$, whilst manual identification resulted in an AUC value of $85.4 \%$. Results show that the effectiveness of distinguishing by a radiologist between benign and malignant solitary pulmonary nodules will increase with the aid of this system (Aoyama et al., 2002).

In relation to the process of computer aided image processing methods different experiences obtained. Al-Fahoum et al proposed a technique for lung image processing with the following stages: segmentation, labeling the regions of interest and extracting diagnostic features of the regions. Study results show that accuracy of radiologist's detection rate for small lung nodules improved $98.13 \%$ by using the computerized image processing technique. It improved physician diagnosis in early stage of lung cancer and increased effectiveness (Al-Fahoum et al., 2014). Sankar in order to detection of lung cancer by image processing in 2000 samples of lung CT images used stages includes, preprocessing, histogram generation, segmentation, and template matching. The results proved that accuracy of lung cancer detection increased with this image processing approach (Sankar and Prabakaran, 2014). About segmentation and thresholding, approaches are different. Usman et al suggested a segmentation technique to remove images noises in lung region. They demonstrated that in the segmentation stage, Marker-Controlled Water-shed Segmentation technique with accuracy $(5.165 \%)$ and quality $(81.835 \%)$ is better than thresholding technique. This technique improved earlier detection of disease and treatment stages; although needs long time for computation in large data sets (Usman et al., 2013). In addition, Sharma et al that developed automatic lung cancer detection system by image processing technique, and used bit plane slicing technique in extraction phase. The result of their study shows almost $80 \%$ accuracy improvements in the physician diagnosis during reading CT images (Sharma and Jindal, 2011).

On classification of normal or abnormal findings or even malignant or benign lesions in image processing, different algorithms or methods can be used. For example, Ada et al used 909 CT images in DIACOM format with 8 bit resolution for their study, proposed a method consists of images collected, pre-processing of images, feature extraction, principle component analysis, and neural network classifier. For classification of the images the first, they extracted 16 features, then the classification of images (normal or abnormal) performed according to those features. For classification of features, they select neural network algorithm because it gives highest rate of correctly classified instances in compare with ZeroR classifier, Naive Bayes classifier and Support Vector Machine classifier (SVM). The findings demonstrated that, the neural network algorithm with $96.04 \%$ (true positive rate) had the best performance $\left(\mathrm{Ada}^{1}, 2013\right)$. In contrast, Arzhaeva et al presented a system to distinguish normal and abnormal tissues by two-class supervised classification method. The analysis was done by means of the multi scale Gaussian filter ban, linear discriminant 
analysis (LDA), and an SVM classifier (i.e. support vector machine) for 44 abnormal and 8 normal cases. The best performance was $0.78 \%$ with an area under the ROC curve, which achieved by the linear discriminant and SVM classifiers. The best performance to differentiate between abnormal and normal tissues was $0.90 \%$ (Arzhaeva et al., 2007).

In addition, Kato et al suggested a bag of features approach for classification of lung disease with diverse tissues. They use intensity descriptor and scale-invariant feature transformation (SIFT) for feature extraction. Finally, in the 1109 ROIs, classification accuracy to 5 different image class was about $92.8 \%$ using this method (Kato et al., 2009). Moreover, Lingayat et al proposed a computer-based algorithm for feature extraction of lung nodules in X-ray images that helps physicians and radiologist's early diagnosis and deffirentiate between benign and malignant tissues. The developed system with this algorithm, acts as a second opinion to the physicians and radiologists. The results show malignant and benign lung tumor's distinguishes has been improved (Lingayat and Tarambale, 2013).

For higher levels of differentiation, Al-Kadi et al applied CE-CT (Contrast Enhanced Computer Tomography) images to tumor stage prediction. Purpose of this study was improving accuracy of differentiate between aggressive (advanced stage) and non-aggressive (early stage) tumors. Images were in DICOM format and differentiated by Box Counting (DBC) algorithm. The accuracy of this system to differentiate between aggressive and non-aggressive tumor was up to $83 \%$. For this reason, system can be recognized for providing the aggressiveness rate for lung tumors (Al-Kadi and Watson, 2008).

\section{Breast Cancer Diagnosis Through Image Processing}

The second most common cancer overall in the world, the most incidence and the first cause of death for women ages 40-55 is breast cancer (Smith et al., 2004; Karabatak and Ince, 2009; Globocan, 2012b; Gucuk and Uyeturk, 2013). According to GLOBOCAN estimates, 52.9\% of 1.67 million new breast cancers were diagnosed in developing countries in 2012 (Globocan, 2012b). breast cancer is the cause of death in $16 \%$ of cancer-related mortalities (Najmabadi et al., 2014).

An important factor to reduce mortality rate and increase long term survival in breast cancer is early detection and effective treatment (Guo, 2010; Choudhari et al., 2012; Tripathy, 2013; Kazerouni et al., 2014; Mohaghegh et al., 2015; Vithana et al., 2015). Also treatment of breast cancer is very expensive, therefore, early detection leads to reduce personal, health and socioeconomical complication (Zadeh et al., 2012; Kulakci et al., 2015). But because of the similarity between normal and cancerous breast tissues, early detection of breast cancer is difficult. To have an accurate diagnosis, physicians have to detect subtle signs of breast lesions (Abdel-Qader et al., 2006). In this regard, digital image processing can be used to distinguish between breast normal tissues and cancerous lesions. For this purpose, in image processing, segmentation phase plays important roles in differentiate between malignant and benign breast cancer (Zadeh et al., 2009)

\section{Experiences in breast cancer diagnosis through image processing}

About the general effect of the technology for diagnosis of breast cancer, note to research of Freer and et al who developed computer aided detection (CAD) to analysis 2,500 mammograms. They surveyed the effect of CAD on the recall rate, positive predictive value for biopsy, cancer detection rate, and stage of malignancies at detection in compare with radiologist performance. By using of this method increase in breast cancer detection and recall rate was $19.5 \%$ and $1.2 \%$. also detection rate of malignancy in early stage increase 5\% (improvement from $73 \%$ to $78 \%$ ). In positive predictive value for biopsy, there was no significant difference between the radiologist and CAD system (Freer and Ulissey, 2001). However, various methods can be selected and used for it based on the experience gained. For instances Nagaraj and his colleagues proposed Effective Statistical Texture Detection algorithm (ESTD) that had four core stages and can be used to MRI, magnetic resonance angiography, and ultrasonography imaging techniques with different visual attributes. The four steps is include de-noising, statistical treshholding, identify suspicious objects with statistical measure and determine selection criteria to selecting suspicious objects. The algorithm applied for 100 mammographies in a database. Evidences have been showed that the proposed algorithm achieved $70 \%$ true result with simulation (Nagaraj et al., 2014). But for mammographic digital images, Mencattini proposed wavelet transform for enhancement and de-noising. This algorithm applied for many mammography images. It is reported that the boundaries of the mass were more differentiable and region of interest could distinguish better. In total, the algorithm had effective performance (Mencattini et al., 2008).

Guzman-Cabrera et al proposed a technique to identify the region of interest in masses and micro-calcifications from tissue by feature extraction through texture analysis in eight-bit gray scale mammography images (benign or malignant); and detects the amount of area that should be identified and extracted (reference gray level). The performance of the algorithm depends on the size of reference area that should be removed after segmentation. As a result, for micro-calcifications when large area was removed correlation coefficient increased, and for masses when small area was removed correlation coefficient increased. This distinguishes between micro-calcifications and masses help physician to have better image analysis and more effective diagnosis (Guzman-Cabrera et al., 2013).

For thermographs also, researchers applied an automated algorithm. The methode involves edge detection, breast boundaries detection, feature extraction, asymmetry description and cumulative histogram. These algorithm used for diagnosis of cancer regions. Results show this method was effective in diagnosing the asymmetric abnormalities (Kapoor et al., 2010). In 
contrast,Abaspur Kazerouni used the principal component analysis (PCA) and the two-dimensional principal component analysis (2D PCA) and the two-directional two-dimensional principal component analysis ((2D) 2PCA) to best analysis of the thermographic images. Also, the support vector machine (SVM) with RBF kernel (radial basis function kernel) used for image retrieval. This approach applied for 400 thermographic images. As the result, the highest precision value was $99.33 \%$ for (2D)2PCA and $90.86 \%$ for 2DPCA and 82.98 for PCA (Kazerouni et al., 2014).

However, other researchers in their time, proposed a novel image detection method for boundary detection of tumors in breast cancer digital mammographies by Neural Network Classifier. This technique use NN classifier with $\mathrm{N}$-ary morphological operator to eliminate the boundary errors. Moreover, for large number of images they used from Gabor filter for elimination of noise and classifying the tissues abnormalities to benign or malignant. This method applied for natural and synthetic $512 \times 512$ pixel 8 -bit grayscale images successfully. It has been reported that diagnosis was improved by this technique (Reddy et al., 2010). In the study of Shanthi and et al, proposed an integration of intuitionistic Fuzzy C-Means clustering and Self Adaptive Resource Allocation Network classifier to classification of breast cancer status according to the mammogram images. This technique applied for 322 normal, benign and malignant images. Proposed system's precision, recall and F-means compared with four classifiers (RBFN, MLP, Navie bayes and C4.5) and at the result the proposed system had the highest precision, recall and $\mathrm{F}$ means for classification of image as normal, benign or malignant. With this system early detection of breast cancers improved (Shanthi and Bhaskaran, 2012). In other research, applied image processing threshold, edge-based and watershed segmentation for mammogram breast cancer images; and test three algorithms with MatLab software. By comparing time consuming and simplicity in these three methods, It was concluded that threshold method was faster than other methods but two other methods had better output images (Alhadidi et al., 2007). In the same study by Shareef applied morphological operation and segmentation watershed transformation to process the images that captured from ultrasound and X-ray mammography devices among women aged between 40-60 years. Similar diagnosis found in the different types of images by using this algorithm. The accuracy of diagnosis was acceptable about $84.84 \%$ (Shareef, 2014).

In conclusion, because, images are essential components in many fields of medicine, especially for the diagnosis process of tumors, technology based approaches such as digital image processing help physicians to have better interpretation, faster detection and increases accuracy and objectivity of diagnosis. In cancer diagnosis and treatment field the goal of digital image processing is to locate and extract meaningful items from images and classify the type and stage of cancer. Looking at the experiences gained will help specialists to choose the appropriate technique for optimization of diagnosis through medical imaging. But it is necessary to emphasize that, although the literature review indicates that digital image processing techniques have an important role in easing and early detection of lung and breast cancers, but this technology cannot replace human judgment and just have an assistant role in judgment for diagnosis and treatment of cancers. These approaches decrease expert's disagreements and lead to better and common understanding of stage of disease.

\section{References}

Abdel-Qader I, Shen L, Jacobs C, et al (2006). Unsupervised detection of suspicious tissue using data modeling and PCA. Int J Biomed Imaging, 2006, 57850

Abdulbaki AS (2012). Skin cancer image segmentation \& detection by using unsupervised neural networks (UNN).

Abramoff MD, Magalhaes PJ, Ram SJ (2004). Image processing with Image. J Bio Int, 11, 36-43.

Al-Fahoum AS, Jaber EB, Al-Jarrah MA (2014). Automated detection of lung cancer using statistical and morphological image processing techniques. J Biomedical Graphics Comput, 4, 33 .

Al-Kadi OS, Watson D (2008). Texture analysis of aggressive and nonaggressive lung tumor CE CT images. Biomedical Engineering, IEEE Transactions On, 55, 1822-30.

Al-Tarawneh MS (2012). Lung cancer detection using image processing techniques. leonardo electronic.J Pract Technol, 11, 147-58.

Alhadidi B, Zu'bi MH, Suleiman HN (2007). Mammogram breast cancer image detection using image processing functions. Informat Technol J, 6, 217-21.

Aoyama M, Li Q, Katsuragawa S, et al (2002). Automated computerized scheme for distinction between benign and malignant solitary pulmonary nodules on chest images. Med Phys, 29, 701-8.

Arzhaeva Y, Prokop M, Tax DM, et al (2007). Computer-aided detection of interstitial abnormalities in chest radiographs using a reference standard based on computed tomography. Med phy, 34, 4798-809.

Azadmanjir Z, Safdari R, Ghazisaeidi M (2015). From self-care for healthy people to self-management for cancer patients with cancer portals. Asian Pac J Cancer Prev, 16, 1321-5.

Bae KT, Kim JS, Na YH, et al (2005). Pulmonary nodules: automated detection on ct images with morphologic matching algorithm-preliminary results 1 . Radiol, 236, 286-93.

Chen S, Zhao M, Wu G, et al (2012). Recent advances in morphological cell image analysis. Comput Math Methods Med, 2012, 101536

Chen Y, Huang X, Shi H, et al (2011). A novel and cost-effective method for early lung cancer detection in immunized serum. Asian Pac J Cancer Prev, 12, 3009-12.

Choudhari G, Swain D, Thakur D, et al (2012). Colorography: an adaptive approach to classify and detect the breast cancer using image processing. Int J Comp Appl, $\mathbf{4 5}$.

Demir C, Yener B (2005). Automated cancer diagnosis based on histopathological images: a systematic survey. Rensselaer Polytechnic Institute Tech Rep [Epub ahead of print].

Dobrescu R, Dobrescu M, Mocanu S, et al (2010). Medical images classification for skin cancer diagnosis based on combined texture and fractal analysis. WISEAS Trans on Biol Biomedicine, 7, 223-32.

Fallahzadeh H, Momayyezi M, Akhundzardeini R, et al (2014). five year survival of women with breast cancer in Yazd. Asian Pac J Cancer Prev, 15, 6597-601.

Freer TW, Ulissey MJ (2001). Screening mammography with 
computer-aided detection: prospective study of 12,860 patients in a community breast center 1. Radiol, 220,781-6.

G AK (2014). Analysis of medical image processing and its applications in healthcare industry Int. J Comput Technol Appl, 5, 851-60.

Ganesan K, Acharya U, Chua CK, et al (2013). Computer-aided breast cancer detection using mammograms: A review. Biomedical Engineering, IEEE Rev In, 6, 77-98.

Gao M, Bridgman P, Kumar S (2003). Computer-aided prostrate cancer diagnosis using image enhancement and jpeg2000. optical science and technology, SPIE's $48^{\text {th }}$ Annual Meeting. Int Society Optics Photonic, 323-34.

Giger ML (2004). Computerized analysis of images in the detection and diagnosis of breast cancer. Semin Ultrasound CT MR, 25, 411-8.

Globocan (2012a). All Cancers (excluding non-melanoma skin cancer) estimated incidence, mortality and prevalence Worldwide in 2012 [Online].

Globocan (2012b). Globocan 2012. all cancers (excluding nonmelanoma skin cancer) estimated incidence, mortality and prevalence Worldwide in 2012 [Online].

Gucuk S, Uyeturk U (2013). Effect of direct education on breast self examination awareness and practice among women in Bolu, Turkey. Asian Pac J Cancer Prev, 14, 7707-11.

Guo Y (2010). Computer-aided detection of breast cancer using ultrasound images. All Graduate Theses Dissertations, 635.

Guzman-Cabrera R, Guzman-Sepulveda J, Torres-Cisneros M, et al (2013). Digital image processing technique for breast cancer detection. Int J Thermophys, 34, 1519-31.

Hasanabadi H, Zabihi M, Mirsharif Q (2014). Detection of pulmonary nodules in CT images using template matching and neural classifier. J Adv Comput Res, 5, 19-28.

He L, Long LR, Antani S, et al (2012). Histology image analysis for carcinoma detection and grading. Comput Method Programs Biom, 107, 538-56.

Jain VK, Vijay R (2013). Lungs cancer detection from mri image using image processing technique. Int J Comput Technol Appl, 14.

Kannadhasan S, Ahamed NB, RajeshBaba M (2013). Cancer diagonsis with the help digital image processing using ZIGBEE Technology. Int J Emerging Trends Electrical Electronics, 1, 8-10.

Kapoor P, Prasad S, Bhayana E (2010). Real time intelligent thermal analysis approach for early diagnosis of breast cancer. Int J Comput Appl, 1, 22-4.

Karabatak M, Ince MC (2009). An expert system for detection of breast cancer based on association rules and neural network. Expert Systems Appl, 36, 3465-9.

Kato N, Fukui M, Isozaki T (2009). Bag-of-features approach for improvement of lung tissue classification in diffuse lung disease. SPIE Medical Imaging. Int Society Optics Photon, [Epub ahead of print].

Kaur J, Garg N, Kaur D (2014). A survey of lung cancer detection techniques on CT scan Images. Int J Scientific Engineering Res, 5, 377-80.

Kazerouni IA, Zadeh HG, Haddadnia J (2014). A novel model for smart breast cancer detection in thermogram images. Asian Pac J Cancer Prev, 15, 10573.

Kekre H, Sarode T, Raut K (2010). Detection of tumor in MRI using vector quantization segmentation. Int J Eng Sci Technol, 2, 3753-7.

Kemal Y, Yucel I, Ekiz K, et al (2014). Elevated serum neutrophil to lymphocyte and platelet to lymphocyte ratios could be useful in lung cancer diagnosis. Asian Pac J Cancer Prev, 15, 2651-4.

Kulakci H, Ayyildiz TK, Yildirim N, et al (2015). Effects of breast cancer fatalism on breast cancer awareness among nursing students in Turkey. Asian Pac J Cancer Prev, 16, 3565.

Lee N, Laine AF, Marquez G, et al (2009). Potential of computeraided diagnosis to improve CT lung cancer screening. Biomedical Engineering, IEEE Rev Biomed Eng, 2, 136-46.

Leela G, Kumari HV (2014). Morphological approach for the detection of brain tumour and cancer Cells. J Electron Comput Eng Res, 2, 7-12.

Lingayat NS, Tarambale MR (2013). A computer based feature extraction of lung nodule in chest $\mathrm{x}$-ray image. Int $J$ Bioscience Biochem Bioinformat, 3, 624-9.

Loukas CG, Wilson GD, Vojnovic B, et al (2003). An image analysis-based approach for automated counting of cancer cell nuclei in tissue sections. Cytometry Part A, 55, 30-42.

Mencattini A, Salmeri M, Lojacono R, et al (2008). Mammographic images enhancement and denoising for breast cancer detection using dyadic wavelet processing. IEEE Trans Med Imaging, 57, 1422-30.

Mohaghegh P, Yavari P, Akbari ME, et al (2015). Associations of demographic and socioeconomic factors with stage at diagnosis of breast cancer. Asian Pac J Cancer Prev, 16, 1627-31.

Mohammadzadeh N, Safdari R (2014). Robotic surgery in cancer care: opportunities and challenges. Asian Pac J Cancer Prev, 15, 1081-3.

Mukti MZR, Ahmed F (2013). Early detection of lung cancer risk using data mining. Asian Pac J Cancer Prev, 14, 595-8.

Nagaraj H, Paga P, Lamichhane K (2014). Early breast cancer detection using statistical parameters. Int J Res Engineer Technolo, 2, 31-6.

Najmabadi KM, Azarkish F, Latifnejadroudsari R, et al (2014). Self-disclosure of breast cancer diagnosis by Iranian women to friends and colleagues. Asian Pac J Cancer Prev, 15, 2879-82.

Patil BG, Jain SN (2014). Cancer cells detection using digital image processing methods. Intl $J$ Latest Trends Engineer Technolo, 3, 45-49.

Patil SN, Kulhalli K, Patil SS (2014). Endometrial cancer detection using image processing techniques. Intl J Software Hardware Res Engineer, 2, 20-3

Polakowski WE, Cournoyer DA, Rogers SK, et al (1997). Computer-aided breast cancer detection and diagnosis of masses using difference of Gaussians and derivative-based feature saliency. IEEE Trans Med Imaging, 16, 811-9.

Rajyalakshmi U, Satya Prasad K, Koteswara Rao S (2014). Tissue processing, staining and image processing of pathological cancer images: A Review. Int J Eng Adv Technol, 3, 10-5.

Ramteke NS, Jain SV (2013). Analysis of skin cancer using fuzzy and wavelet technique-review \& proposed new algorithm. Int J Engineer Trend Technol, 4.

Reddy L, Reddy R, Madhu C, et al (2010). A novel image segmentation technique for detection of breast cancer. Int $J$ Inf Technol Knowl Manag, 2, 201-4.

Reményi A, Szenasi S, Bandi I, et al (2011). Parallel biomedical image processing with GPGPUs in cancer research. Logistics and Industrial Informatics (LINDI), $20113^{\text {rd }}$ IEEE international symposium on, IEEE, 245-8.

Sampat MP, Markey MK, Bovik AC (2005). Computer-aided detection and diagnosis in mammography. Handbook Image Video Proces, 2, 1195-217.

Sankar K, Prabakaran M (2014). An efficient template matching algorithm for lung cancer detection using multi resolution histogram based image segmentation. Int $J$ Enhanced Res in Sci Technolo Engineer, 3, 71-5.

Santosh A, Sadashivappa G (2014). Skin cancer detection and diagnosis using image processing and Implementation 
using neural networks and ABCD parameters. Int J Elect, Communic Instrument Eng Res Develop, 4, 85-96.

Shanthi S, Bhaskaran VM (2012). Computer aided system for detection and classification of breast cancer. Int J Inf Technol Control Autom, 2, 87-98.

Shareef SR (2014). Breast cancer detection based on watershed transformation. Int J Computer Sci, 11, 237-45.

Sharma D, Jindal G (2011). Identifying lung cancer using image processing techniques. Int Conf Comput Techniques Artificial Intelligence, 2011, 872-80.

Shridhar K, Dey S, Bhan CM, et al (2015). Cancer detection rates in a population-based, opportunistic screening model, New Delhi, India. Asian Pac J Cancer Prev, 16.

Shriwas RS, Dikondawar AD (2015). Lung cancer detection and prediction by using neural network. Int $J$ Electronics Communicat, 3, 17-21.

Smith AP, Hall PA, Marcello DM (2004). Emerging technologies in breast cancer detection. Radiol Manage, 26, 16-27.

Strickland RN 2002. Image-processing techniques for tumor detection, CRC Press.

Sundari KS, Vanaselvi P, Vishakai T (2014). Diagnosis of rectal cancer through images. Int $J$ Advanced Networking and Applications, 6, 2329-33.

Upadhyay Y, Wasson V (2014). Analysis of Liver MR Images for Cancer Detection using Genetic Algorithm. Int J Engineer Res General Sci, 2, 730-7.

Usman M, Shoaib M, Rahal M(2013). Multi-resolution analysis technique for lung cancer detection in computed tomograpic images. Session 4AK, 1455.

Vemuri RC, Jarecha R, Hwi KK, et al (2014). Importance of volumetric measurement processes in oncology imaging trials for screening and evaluation of tumors as per response evaluation criteria in solid tumors. Asian Pac J Cancer Prev, 15, 2375.

Vithana PC, Ariyaratne M, Jayawardana P (2015). Educational intervention on breast cancer early detection: effectiveness among target group women in the district of gampaha, sri lanka. Asian Pac J Cancer Prev, 16, 2547-53.

Zadeh HG, Haddadnia J, Hashemian M, et al (2012). Diagnosis of breast cancer using a combination of genetic algorithm and artificial neural network in medical infrared thermal imaging. Iranian J Med Phy, 9, 265-74.

Zadeh HG, Janianpour S, Haddadnia J (2009). Recognition and classification of the cancer cells by using image processing and Lab VIEW. Int J Computer Theory Eng, [Epub ahead of print].

Zahir ST, Mirtalebi M (2012). Survival of patients with lung cancer, Yazd, Iran. Asian Pac J Cancer Prev, 13, 4387-91.

Zhang H, Fritts JE, Goldman SA (2008). Image segmentation evaluation: A survey of unsupervised methods. Computer Vision Image Understand, 110, 260-80. 